\title{
Lipid Nanoparticles Delivery of CRISPR/Cas9 Targeting PCSK9 and ANGTPL3 as New Therapeutic Gene Editing Modalities for Potential Long-Lasting Treatment Of Dyslipidemia
}

\author{
Ramidha Syaharani ${ }^{a}$, Fauziah Adhima ${ }^{a}$, Sarah Nia Amru ${ }^{a}$, Reny I'tishom ${ }^{\text {b** }}$ \\ ${ }^{a}$ Medical Program, Faculty of Medicine, Universitas Airlangga 60132, Surabaya, Indonesia \\ ${ }^{b}$ Department of Biomedical Sciences, Faculty of Medicine, Universitas Airlangga 60132, Surabaya, Indonesia
}

Corresponding Author : ritishom@fk.unair.ac.id

\begin{abstract}
Dyslipidemia is the main risk factor for atherosclerosis leading to cardiovascular disease, one of the important health problems in the Asia Pacific region. Several dyslipidemia treatment modalities such as statins and monoclonal antibodies were considered less effective both from the aspect of cost or toxic side effect. The aim of this study is to describe the potential of lipid nanoparticle-mediated efficient delivery of clustered regularly interspaced short palindromic repeat (CRISPR) associated protein 9 (CRISPR/Cas9) targeting proprotein convertase subtilisin/kexin type 9 (PCSK9) and angiopoietin-like protein 3 (ANGPTL3) as new therapeutic genome editing modalities for potential long-lasting treatment of dyslipidemia. The method used in this study is to explore the literature in the form of systematic reviews, metaanalysis, and randomized control trials (RCTs) through several search engines such as Sciencedirect, Pubmed, and Google Schoolar in the last 10 years. The outcome of this study is to review the effectiveness of PCSK9 and ANGPTL3 inhibition in lowering cholesterol levels making both genes as a major therapeutic target for the treatment of dyslipidemia. Currently, an efficient way to permanently inhibit both genes has been developed using CRISPR-Cas9 genome editing delivered by lipid nanoparticles (LNPs), the most effective non-viral delivery modalities that work specifically on the liver. A single administration of LNPs-CRISPR/Cas9 in mice produced undetectable PCSK9 serum levels more than $80 \%$ and a drop of total cholesterol by 35\%-40\%. Meanwhile, CRISPR/Cas9 targeting ANGPTL3 resulted in a greater decrease in triglycerides on 7 day post-treatment. As a conclusion, genome editing therapy based on CRISPR/Cas9 lipid nanoparticles targeting PCSK9 and ANGPTL3 is promising for the treatment of dyslipidemia.
\end{abstract}

Keywords: ANGPTL3, CRISPR/Cas9, dyslipidemia, Lipid Nanoparticles(LNPs), PCSK9

\section{Introduction}

Dyslipidemia is a major risk factor for atherosclerosis which can cause ischemia in the brain, heart, or legs and induce cardiovascular disease, one of non-communicable disease which is an important health problem in the Asia Pacific Region.[1,2] Dyslipidemia is characterized by high levels of low-density lipoprotein cholesterol (LDL-C), total cholesterol (TC), and triglycerides (TG), or low levels of high-density lipoprotein cholesterol (HDL-C) in the blood.[3] In Asian countries such as China, dyslipidemia is the second most common metabolic syndrome component after hypertension.[4] According to the WHO statistics, the prevalence of dyslipidemia in adults aged $>25$ years in Indonesia, on the basis of total cholesterol concentration $160 \mathrm{mg} / \mathrm{dL}$, was about 36\%.[5] A study in a small population showed that the prevalence of dyslipidemia in all ethnic groups in Indonesia was between $9.0 \%$ and $25 \%$ with Minangkabau ethnic group as the highest total plasma cholesterol and plasma LDL-C and Sundanese as the lowest plasma HDL-C.[6] 
Hyperlipidemia is often a lifelong disease process that can actually be managed well. However, if hyperlipidemia is left untreated, the disease progresses progressively and can lead to severe underlying vascular disease processes, which can prove fatal.[7] Various complications of untreated hyperlipidemia include coronary artery disease, peripheral artery disease, cerebrovascular accidents, aneurysms, type II diabetes, high blood pressure, and even death. So that effective and efficient treatment of hyperlipidemia to prevent the progression of this disease is certainly very necessary.[8] Based on clinical outcome studies, LDL$\mathrm{C}$ levels have been shown to be closely related to cardiovascular disease[9], therefore LDL-C is the main target in the management of dyslipidemia to prevent cardiovascular disease.[10] In addition, Serine protease proprotein subtilisin/kexin convertase type 9 (PCSK9), a major regulator of LDL metabolism which can increase LDL receptor (LDLR) degradation, is also a potential target for dyslipidemia therapy.[11] [12] When PCSK9 is inhibited, LDLR expression and activity are increased, resulting in a decrease in LDL-C levels.[13] In contrast to angiopoietin-like 3 (ANGPTL3), which has been shown to inhibit the activity of Lipoprotein lipase (LPL) and endothelial lipase (EL) resulting in an increase in plasma concentrations of TG, LDL-C and HDL-C.[14] Thus, inhibition of ANGPTL3 is also considered a promising pharmacological target for the treatment of dyslipidemia.[15]

Until now, various drugs and therapies for dyslipidemia to reduce LDL-C levels have been developed. There are several lipid lowering agents, such as statins, nicotinic acids, fibrates, and bile acid sequestrants,[9] but statins are the preferred medical treatment for lowering LDL-C agent to achieve the optimum target of LDL-C levels.[10] However, despite receiving intensive statin therapy, many patients cannot achieve optimum lipid levels.[16] On the other hand, high-dose statins can increase the incidence and severity of side effects such as muscle toxicity.[17] Alternatively, inhibition of PCSK9 with monoclonal antibodies (mAbs) has been approved as a second-line treatment in at-risk patients who cannot achieve optimal target LDL-C levels despite maximally tolerated statin therapy or for patients with statin intolerance.[18,19] However, mAbs show a relatively short half-life in vivo and should be applied frequently and highly costs if given long term.[13]

Gene editing technology is a potentially powerful therapeutic tool that can precisely and efficiently trim, cut, replace, or insert DNA or RNA sequences.[20,21] One of the gene editing technologies that has been developed and won the 2020 Nobel Prize in Chemistry is clustered regularly interspaced short palindromic repeat (CRISPR) associated protein 9 (CRISPR/Cas9).[21] It consists of two components that can simplify genome editing procedures, namely using single-guide RNA (sgRNA) to recognize target DNA, and Cas9 nuclease activity to introduce site-specific double-strand breaks (DSBs) at the target gene loci.[22] Recently, Cas 9 nuclease and sgRNA are delivered to target cells via nonviral nanocarriers, lipid nanoparticles (LNPs), who have a favorable safety profile and have been developed for delivery of Cas9 plasmid DNA, mRNA, and ribonucleoproteins (RNPs).[23-25] Several studies reported that the use of LNPs has successfully delivered CRISPR-Cas9 in both the RNP and mRNA formats.[26-29] mRNA delivery considered very promising in in vivo genome editing applications because of a transient and non-integrated Cas 9 expression feature.

Lately, the use of bioreducible LNPs for the codelivery of Cas9 mRNA and gRNA, demonstrated highly efficient in vitro genome editing, as well as rapid knockdown of the PCSK9 cholesterol-regulating gene in vivo, highlighting the potential of this delivery approach.[30] In addition, a lipid nanoparticle delivery platform carrying Cas9 mRNA and guide RNA for CRISPR/Cas9-based genome editing of ANGPTL3 in vivo, was also reported to mediate ANGPTL3 gene knockdown specifically and efficiently in theliver of wildtype C57BL/ 6 mice, resulting in profound reductions in serum ANGPTL3 protein, LDL-C, and TG levels.[20] Therefore, this study aims to describe the potential of lipid nanoparticle-mediated efficient delivery of CRISPR/Cas9 Genome Editing as new therapeutic modalities to knockout genes related to cardiovascular disease, PCSK9 and ANGPLT3, as potential long-lasting treatment of dyslipidemia. 


\section{Methods}

The method used in this study is to explore the literature in the form of systematic reviews, meta-analysis, and randomized control trials (RCTs) with a level of evidence 1A-2C through several search engines such as Sciencedirect, Pubmed, and Google Schoolar using the keywords "ANGPTL3", "CRISPR/Cas9", "dyslipidemia", "Lipid nanoparticles(LNPs)", and "PCSK9". To get specific search results, "Mesh Database" and Boolean Operators ("AND", "OR", "NOT") are used when searching literature. The literature search was limited to studies published in the last 10 years and available in English or Indonesian.

\section{Results and Discussion}

\subsection{The Role of PCSK9 and Its Inhibition as a Management of Dyslipidemia}

Dyslipidemia is a condition of lipid imbalance characterized by high levels of cholesterol, TG, LDL-C, and low levels of HDL. The main focus of lipid treatment is to prevent complications from atherosclerosis, which can lead to many cardiovascular disease as an important risk factor of dyslipidemia.[31] LDL-C has been recommended as the main therapeutic target in cardiovascular disease.[32]

Recent studies have shown that PCSK9 plays a significant role in the metabolism of triglyceride-rich lipoprotein through its interaction with the LDLR.[33] PCSK9 is a member of a serine protease that has the ability to hydrolyze peptide bonds for activation.[34] PCSK9 is known to be a major regulator of LDL metabolism.[11] LDLR is reduced by PCSK9 in hepatocytes through metabolism and degradation.[34]

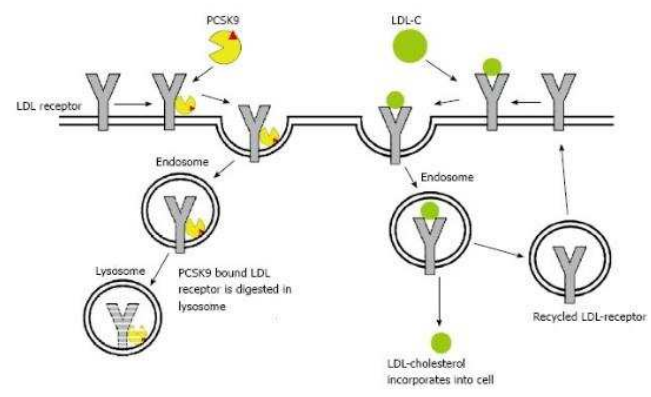

Figure 1: Mechanism and role of PCK9 in low-density lipoprotein-cholesterol (LDL-C) metabolism.[34]

Under normal circumstances, the LDL-C complex and LDLR are endocytosed by the endosome. The affinity of LDL-C to LDLR can be reduced by the acidic environment of the endosome which can trigger the recycling of LDL-C to return to the plasma membrane. The recycling of LDLR is inhibited by the binding of PCSK9 on the hepatocyte cell surface. LDLR is then directed to lysosomes for degradation thereby reducing the number of LDLR on the cell surface.[34] The mechanism of PCSK9 inhibition is expected to increase the amount of LDLR available on the cell surface and increase the absorption of LDL-C into cells so that it can reduce LDL-C levels in the blood circulation.[33]

PCSK9 is expressed both intracellularly and in the circulation. There are several targets for PCSK9 inhibition. These modalities include inhibition of production by gene silencing through antisense oligonucleotides or small interfering RNAs; prevention of binding of PCSK9 to LDLR using monoclonal antibodies, mimetic peptides or adnectin, inhibition of PCSK autocatalytic sites and epidermal growth factorlike repeat A (EGF-A).[34,35] Recent developments have shown that there is a more efficient way to 
permanently inhibit PCSK9. This method is carried out through an in vivo mechanism using the CRISPR/Cas9 system.[36,37]

\subsection{The Role of ANGPTL3 and Its Inhibition as a Management of Dyslipidemia}

ANGPTL3 is a member of the angiopoietin-like protein (ANGPTLs) family which is expressed in the liver and has been recognized as an important regulator of lipid metabolism. ANGPTLs consist of 8 members and all play an important role in plasma lipid metabolism. Compared to the eight types of ANGPTLs, ANGPTL3 has recently attracted the attention of researchers in recent years.[15] Plasma levels of TG and LDL-C can be increased by increasing ANGPTL3. Therefore, it is possible to reduce the levels of TG, LDL-C, and atherosclerotic lesion size by inhibiting ANGPTL3. This inhibitory mechanism is expected to reduce the risk of dyslipidemia and cardiovascular disease.[15,38]

The mechanism of ANGPTL3 inhibition works by activating lipoprotein lipase (LPL) in peripheral tissues which plays a role in hydrolyzing TG carried by VLDL and chylomicron (CM) particles in the circulation. In addition, ANGPTL3 also activates endothelial lipase (EL) and prevents the secretion of triglyceride-rich lipoproteins (TLRs) by the liver. All of these mechanisms result in decrease of TG and total cholesterol level. $[33,39]$ In addition to the previous therapy for dyslipidemia, which more focused on lowering LDL-C levels, there are currently pharmacological approaches targeting other lipoproteins, one of which is TG.[40]

Compared to PCSK9 which is known to be more potent in lowering LDL-C levels in the blood, ANGPTL3 targeted therapy is known to be more potent in lowering TG levels. Interestingly, the inhibition of both ANGPTL3 and PCSK9 did not show a synergistic or additive effect. This can be influenced by differences in lipid metabolism pathways between species.[41]Several ways to inhibit ANGPTL3 have been developed. Among them are inhibiting antibodies, genome editing to trigger loss of function mutations at the gene level and antisense oligonucleotides to inhibit the translation of ANGPTL3 messenger RNA. Currently, a way to permanently inhibit ANGPTL3 has been developed, namely through in vivo gene editing with CRISPR/Cas9 technology.[41]

\subsection{CRISPR/Cas9}

The protein nuclease system (CRISPR/Cas9) is a genome editing tool by removing or adding DNA sequences for various biomedical applications. The CRISPR/Cas9 system as a ribonucleoprotein complex (RNP) consisting of the Cas9 protein and a sgRNA.[36,42] Cas9 can cleave DNA sequences through guidance by sgRNA which can identify specific target genomic loci. The CRISPR/Cas9 system has been shown to be successful in inhibiting PCSK9 and recently completed a phase III clinical trial on the treatment of hyperlipidemia and successfully treat dyslipidemia in adult mice.[20,37,43,44] Compared with conventional antisense oligonucleotide (ASO) or temporary antibody therapy, the Cas9 CRISPR system can induce permanent loss function which can produce long-term therapeutic effects and can work more safely, specifically, and efficiently.[20]

Delivery modalities in CRISPR/Cas9 include non-viral vectors, viral vectors, and physical delivery. This viral modality in delivering CRISPR/Cas9 is limited and has a minimal load capacity.[45] Meanwhile, physical delivery such as electroporation is time-consuming and labor-intensive, so its application is only to a small number of species due to its limitations. Recently, non-viral nanodelivery is known to efficiently deliver CRISPR/Cas9 in vitro and in vivo.[45] The challenge for CRISPR/Cas9 in entering cells is due to the large size of the protein molecule (cas9 genetic size $-4.5 \mathrm{~kb}$ ) and poor stability. The nanocarrier is the ideal delivery 
modality for CRISPR/Cas9. Some of the nanomaterials that have been developed include lipid LNPs, cationic polymers, vesicles, and gold nanoparticles.[45-47]

One of the advantages of LNPs over others is that they are said to be safer for delivery of Cas9 plasmid DNA, mRNA, and RNPs. LNPs are said to be the most efficacious class of RNA delivery carriers in humans and experimental animals. LNPs itself consist of dendrimer lipid nanoparticles (DLNPs), stable nucleic acid lipid particles (SNALPs), and lipid-like nanoparticles (LLNPs). CRISPR/Cas9 is known to be applicable to three different types of LNPs which allows modified LNPs to be able to rapidly edit cell DNA in different tissues.[20,23,24,25]

\subsection{CRISPR/Cas9 lipid nanoparticles (LNPs) as a Promising Therapy of Dyslipidemia}

\subsubsection{Lipid nanoparticles delivery of CRISPR/Cas9 development}

A hindrance in CRISPR/Cas9 mediated genome editing is ineffective delivery of genome editing proteins caused by instability and low membrane permeability. This caused by Cas 9 protein and sgRNA is not naturally exist within mammalians and cell permeable.[26,30] Widely used viral vectors recently arise some concerns such as tumorigenesis, mutagenesis, immunogenicity, restricted package capacity, large size of Cas 9 and off target effects. As there is many limitations within viral delivery, the delivery has shifted to non-viral delivery with advantage such as safety, simplicity, and flexibility.[48,49]

In most recent study, LNPs was recognized as currently most effective approach of RNA delivery carriers in preclinical models also in human.[50,51] LNPs delivery is combining negatively charged nucleic acids with positively charged lipids through electrostatic interactions forming LNPs. The precedence of cationic liposome is the interaction with negatively charged cell membrane resulting in easier nucleic acids encapsulating process. LNPs preserve nucleic acids from nuclease and mainly infiltrate target cells by means of endocytosis or macro-pinocytosis. Common available lipids, Lipofectamine 3000 and RNAiMAX, may be immediately used to deliver RNPs to carry out gene editing.[26,52]

But, it is commonly believed that LNPs preferably modified for Cas9 mRNA-sgRNA RNPs because consist of complexation of cationic lipids and anionic RNPs.[53] A study was conducted by Wang et al. using 12 bioreducible cationic lipids combined with anionic Cas9: sgRNA complexes to facilitates endosome escape of protein. Endosomal escape further contributes to delivering Cas9 into the nucleus for genome editing. Endosomal escape was also improved by integrating reducible disulfide bonds towards lipid hydrophobic tail.[21] Five out of 12 lipids success deliver Cas9/sgRNA complex with effectivity more than $50 \%$ in rodents brain.[26] Lipid mediated Cas9mRNA delivery has proven that it reaches target more accurately than lentivirus-mediated Cas9 delivery.[54] Also Cas9 mRNA method claims reducing mutagenesis, transient effects, reaching target cells more accurately and reduced complexity compare to Cas 9 plasmid.[53]

Liu et al. demonstrated 7 of 32 lipids efficiently delivering Cas 9 mRNA-sgRNA to lowering green fluorescent protein (GFP) expression. BAMEA-O16B, a leading lipid, appears promising with efficieny $90 \%$ knocks out GFP expression of HEK (human embryonic kidney) cells. The process occurs during 24h post administration. Further the study selected HPV18 an essential gene that triggers cervical cancer as a target cells. BAMEA-O16B/Cas9 mRNA/sgHPV18 was significantly prohibited HeLa growth rather than just combination of Cas 9 mRNA/sgHPV18.[30]

In the development of therapeutic gene editing to medicate amyloidosis, more than $>97 \%$ breakdown of serum transthyretin (TTR) levels as target protein for 12 months long in rat model was achieved by lipid nanoparticles based CRISPR/Cas9 delivery. The editing capacity is cumulative adhering multiple LNPS doses and biodegradable lipid and CRISPR/Cas9 element are temporary and well tolerated.[27] The discovery of 
CRISPR/Cas9 lipid nanoparticles based delivery in cancer therapies are promising. One single intracerebral injection of CRISPR/Cas9 able to editing $70 \%$ gene against polo like kinase 1(PLK1) in orthotopic glioblastoma and intraperitoneal injections able to editing $80 \%$ gene against PLK1 in ovarian tumors.[55] Dopamine signaling and relieving Parkinson's disease related symptoms effect was increased by single injection of lipid nanoparticles based CRISPR/Cas9 delivery into brain rich dopaminergic neuron area.[26] Modification of LNPs, DLNPs, capable to recover dystrophin expression in duchenne muscular dystrophy (DMD) mice.[36]

Despite many promising progress in LNPs delivery of CRISPR/Cas9, the safe and effective delivery has become attention that needs to considered in future research. A research by Schaefer et al using CRISPR/Cas9 for sight restoration in blind $\mathrm{rd} 1$ mice, shows that there is unexpectedly high number of single-nucleotide variants (SNV) denoting high number of mutations in a mouse model of gene therapy. Future studies needs to increase the accuracy of CRISPR/Cas9 and decrease the risk of mutations.[56,57]

\subsubsection{Lipid nanoparticles delivery of CRISPR/Cas9 targeting PCSK9 as a Therapy of Dyslipidemia}

CRISPR/Cas9 targeting PCSK9 became a new trend. PCSK9 will stimulates lysosomal degradation of LDLR, and PCSK9 function loss will results in low levels of LDL-C.[58] CRISPR/Cas9 expected to treats hyperlipidemia and it has ability of modifying genome to permanently decrease cholesterol levels.[59,60] The development started with utilization CRISPR/Cas9 adenovirus delivery in murine livers. The study shows that by disrupting PCSK9 genes, cholesterol level is reduced. PCSK9 blood levels also decrease significantly in mice with humanized livers indicating it safety and effectiveness in reducing human PCSK9 levels.[61] But as mentioned before, despite viral vectors have delivery efficiency, their biosecurity is still maintained as problem. Adenovirus also can provoke deleterious immune response due to its prolonged expression. Mutagenesis can be produced by Adenovirus in just 3 to 4 days. A study by Chadwick et al. used PCSK9 base editing method rather than breaking double strand that could result in mutagenesis. Base editor delivered into mice could introduce site-spesific nonsense mutations towards PCSK9 gene. The PCSK9 protein levels reduced by more than $50 \%$ and plasma cholesterol levels reduced by $30 \%$. Off-target mutagenesis and cytosine-to-thymine edits cannot be found. The result shows promising possibility prolonged and lifelong reduction of dyslipidemia as cardiovascular risk.[62]

The development shifts to use of LNPs in recent research.[54] The intravenous injection of BAMEA016B/Cas9 mRNA-sgRNA has effectively decrease $20 \%$ of PCSK9 serum concentration in treated mouse.[30] Yin et al. developed LNPs delivery of CRISPR/Cas9 with a purpose to inhibiting PCSK9 gene for hypercholesterolemia treatment. The study shows that a single administration of LNPs-CRISPR/Cas9 in mice produced undetectable PCSK9 serum levels, more than $80 \%$ editing in the liver and a drop of total cholesterol by $35 \%-40 \%$.[54] Study was proven that loss of function mutations in PCSK9 were connected with 15-28\% reduction of LDL-C even in patients that cannot be treated with statin.[63] 

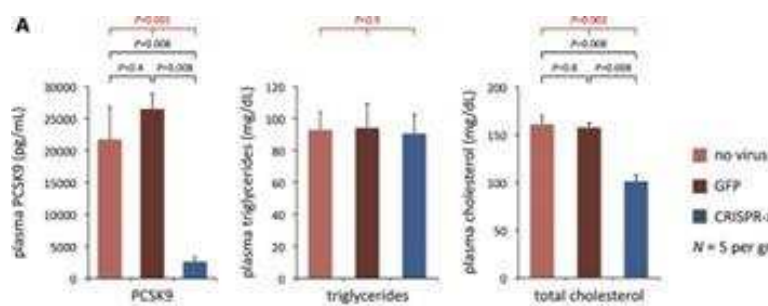

ant

a crisprofesta

$N=5$ per eroup
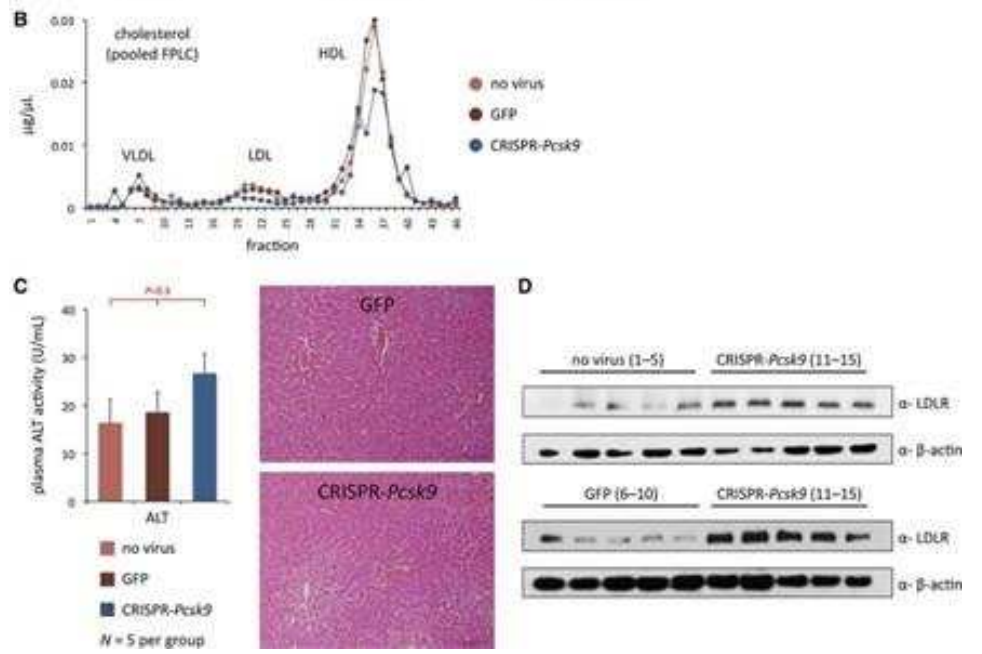

Figure 2: CRISPR/Cas9 delivered by lipid nanoparticles in mice. CRISPR/Cas9 effect shown on PCSK9 serum level, triglycerides, total cholesterol, and ALT. In CRISPR/Cas9 group, plasma cholesterol level reduced up to $35-40 \%$ and no significant differences in triglycerides between no virus, GFP, and CRISPR/Cas9 group.[61]

\subsubsection{Lipid nanoparticles delivery of CRISPR/Cas9 targeting ANGPTL3 as a Therapy of Dyslipidemia}

ANGPTL3 gene was considered as another target of dyslipidemia therapy through CRISPR/Cas9 gene editing. ANGPTL3 considers as an ideal target for gene editing because natural loss of function mutations in ANGPTL3 preserve the body against risk of coronary artery disease without developing serious side effect either in homzygous or compound heterozygous form. The second argument, ANGPTLs expressed by liver hepatocytes and it is secreted into the bloodstream marking its accessibility for various deliver methods. Inactivating mutations, an easier method of gene editing, is the only requisite of ANGPTL3 inhibition.[64]

Chadwick et al conducted the first study using CRISPR/Cas9 base editing to target ANGPTL3. A variation of CRISPR/Cas9 genome editing method called base editing used as in vivo method to promote loss of function mutations into ANGPTL3 in a study by Chadwick et al., BE3-ANGPTL3 injected using adenoviral vectors resulted a median editing rate of 35\% at 7th day. CRISPR/Cas9 targeting ANGPTL3 resulted in greater decrease in triglycerides on 7 day post-treatment rather than CRISPR/Cas9 targeting PCSK9. BE3ANGPTL3 reduced TG (56\%) and cholesterol (51\%) compared to BE3-control in hyperlipidemic LDLRknockout mice. Thus in vivo base editing of ANGPTL3 established as a potential strategy to treat patients with atherogenic dyslipidemia.[65] 


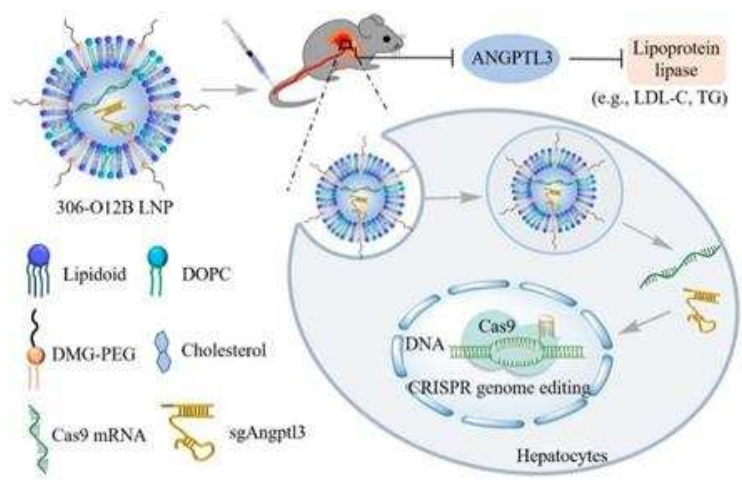

Figure 3: CRISPR/Cas9 delivered by lipid nanoparticles illustration to target ANGPTL3.[20]

The newly founding delivery method by nanoparticles, CRISPR/Cas9 delivered by LNPs able to editing ANGPTL3 gene in the liver of wild-type C57BL/6 mice inducing lowering in serum ANGPTL3 protein $(65,2 \%)$, LDL-C $(56,8 \%)$, and TG $(29,4 \%)$ levels. The gene editing effect lasts stable at least 100 days after a $3 \mathrm{mg} / \mathrm{kg}$ single dose administration. But continued observation proves that the genomic editing effect could still be detected up to 150 day after single injection. No off target mutagenesis found at 9 predicted sites and liver toxicity is not found. The study showing 306-O12B, synthetic and bioreducible lipidoid, is more effective than MC-3 LNPS (gold standard LNPs approved by FDA).[20] Although there is some issues to be addresed in preclinical animal models such as it needs more study about off-target mutagenesis, CRISPR/Cas9 delivery through lipid nanoparticles is a promising therapy of dyslipidemia.

\section{Conclusions}

As a therapy for dyslipidemia, CRISPR/Cas9 lipid nanoparticle-based delivery showed results in lowering LDL-C by targeting PCSK9 and showed greater reduction in triglycerides by targeting ANGPTL3 rather than PCSK9. Thus, genome editing therapy based on CRISPR/Cas9 lipid nanoparticles targeting PCSK9 and ANGPTL3 is promising for the treatment of dyslipidemia. $[30,54,62,63,65]$ Despite many promising progress in lipid nanoparticles delivery of CRISPR/Cas9, future studies needs to considered about the safety and effectiveness of lipid nanoparticles delivery of CRISPR/Cas9.

\section{Acknowledgements}

Gratitude is due to Dr. Reny I'tishom S.Pi, M.Si as our supervisor for his assistance during the preparation of this manuscript.

\section{References}

1. Reid CM, Yan B, Wan Ahmad WA, Bang LH, Hian SK, Chua T, et al. The Asia-Pacific Evaluation of Cardiovascular Therapies (ASPECT) Collaboration - Improving the quality of cardiovascular care in the Asia Pacific Region. Int J Cardiol 2014;172(1):72-5. https://doi.org/10.1016/j.ijcard.2013.12.030

2. Lin CF, Chang YH, Chien SC, Lin YH, Yeh HY. Epidemiology of Dyslipidemia in the Asia Pacific Region. Int. J. Gerontol.2018;12(1):2-6. https://doi.org/10.1016/j.ijge.2018.02.010 
3. Kinoshita M, Yokote K, Arai H, Iida M, Ishigaki Y, Ishibashi S, et al. Japan Atherosclerosis Society (JAS) guidelines for prevention of atherosclerotic cardiovascular diseases 2017. J Atheroscler Thromb 2018;25(9):846-984. https://doi.org/10.5551/jat.GL2017

4. Lan Y, Mai Z, Zhou S, Liu Y, Li S, Zhao Z, et al. Prevalence of metabolic syndrome in China: An up-dated cross-sectional study. PLoS One 2018;13(4). https://doi.org/10.1371/journal.pone.0196012

5. World Health Organization. Global Health Observatory data repository. 2013. http://apps.who.int/gho/data/view.main.2570?lang1/4en

6. Hatma RD. Lipid Profiles Among Diverse Ethnic Groups in Indonesia. 2011. http://www.inaactamedica.org/archives/2011/21339539.pdf

7. Vallejo-Vaz A, Robertson M, Catapano A, Watts G, Kastelein J, Packard C, et al. LDL-Cholesterol Lowering for the Primary Prevention of Cardiovascular Disease Among Men with Primary Elevations of LDL-Cholesterol Levels of $190 \mathrm{mg} / \mathrm{dL}$ or Above: Analyses from the WOSCOPS 5-year Randomised Trial and 20-year Observational Follow-Up. Circulation 2017;136(20):1878-91. https://doi.org/10.1161/CIRCULATIONAHA.117.027966

8. Ford I, Murray H, McCowan C, Packard CJ. Long-term safety and efficacy of lowering low-density lipoprotein cholesterol with statin therapy 20-year follow-up of west of Scotland coronary prevention study. Circulation 2016;133(11):1073-80. https://doi.org/10.1161/CIRCULATIONAHA.115.019014

9. Nelson RH. Hyperlipidemia as a Risk Factor for Cardiovascular Disease. Prim Care - Clin Off Pract 2013;40(1):195-211. https://doi.org/10.1016/j.pop.2012.11.003

10. Erwinanto, Santoso A, Putranto JN, Tedjasukmana P, Suryawan R, Rifqi S, et al. Pedoman Tatalaksana Dislipidemia PERKI 2013. Indones J Cardiol 2013;34(4):245-70.https://doi.org/10.30701/ijc.v34i4.385

11. Shapiro MD, Tavori H, Fazio S. PCSK9 from basic science discoveries to clinical trials. Circ Res 2018;122(10):1420-38. https://doi.org/10.1161/CIRCRESAHA.118.311227

12. Urban D, Pöss J, Böhm M, Laufs U. Targeting the Proprotein Convertase Subtilisin / Kexin Type 9 for the Treatment of Dyslipidemia and Atherosclerosis. J Am Coll Cardiol 2013;62(16):1401-8. https://doi.org/10.1016/j.jacc.2013.07.056

13. Landlinger C, Pouwer MG, Juno C, Van Der Hoorn JWA, Pieterman EJ, Jukema JW, et al. The AT04A vaccine against proprotein convertase subtilisin/kexin type 9 reduces total cholesterol, vascular inflammation, and atherosclerosis in APOE*3Leiden.CETP mice. Eur Heart J 2017;38(32):2499-507. https://doi.org/10.1093/eurheartj/ehx260

14. Ahmad Z, Banerjee P, Hamon S, Chan KC, Bouzelmat A, Sasiela WJ, et al. Inhibition of Angiopoietin-Like Protein 3 with a Monoclonal Antibody Reduces Triglycerides in Hypertriglyceridemia. Circulation 2019;140(6):470-86. https://doi.org/10.1161/CIRCULATIONAHA.118.039107

15. Su X, Peng DQ. New insights into ANGPLT3 in controlling lipoprotein metabolism and risk of cardiovascular diseases. Lipids Health Dis 2018;17(1):1-9. https://doi.org/10.1186/s12944-018-0659-y

16. Koskinas KC, Siontis GCM, Piccolo R, Windecker S, Mavridis D, Raber L, et al. Effect of statins and non-statin LDLlowering medications on cardiovascular outcomes in secondary prevention : a meta-analysis of randomized trials. Eur Heart $\mathrm{J}$ 2018;39:1172-80. https://doi.org/10.1093/eurheartj/ehx566

17. Desai CS, Martin SS, Blumenthal RS. Non-cardiovascular effects associated with statins. BMJ 2014;349:1-10. https://doi.org/10.1136/bmj.g3743

18. Catapano AL, Graham I, De Backer G, Wiklund O, Chapman MJ, Drexel H, et al. 2016 ESC/EAS Guidelines for the Management of Dyslipidaemias: The Task Force for the Management of Dyslipidaemias of the European Society of Cardiology (ESC) and European Atherosclerosis Society (EAS) Developed with the special contribution of the Europea. Atherosclerosis 2016;253:281-344. https://doi.org/10.1016/j.atherosclerosis.2016.08.018

19. Lloyd-Jones DM, Morris PB, Ballantyne CM, Birtcher KK, Daly DD, Depalma SM, et al. 2016 ACC Expert Consensus Decision Pathway on the Role of Non-Statin Therapies for LDL-Cholesterol Lowering in the Management of Atherosclerotic Cardiovascular Disease Risk A Report of the American College of Cardiology Task Force on Clinical Expert Conse. J Am Coll Cardiol 2016;68(1):92-125. https://doi.org/10.1016/j.jacc.2016.03.519

20. Qiu M, Glass Z, Chen J, Haas M, Jin X, Zhao X, et al. Lipid nanoparticle-mediated codelivery of Cas9 mRNA and singleguide RNA achieves liver-specific in vivo genome editing of \&lt;em\&gt;ANGPTL3\&lt;/em\&gt; Proc Natl Acad Sci [Internet] 2021;118(10):e2020401118. Available from: http://www.pnas.org/content/118/10/e2020401118.abstract https://doi.org/10.1073/pnas.2020401118

21. Duan L, Ouyang K, Xu X, Xu L, Wen C, Zhou X, et al. Nanoparticle Delivery of CRISPR/Cas9 for Genome Editing [Internet]. Front. Genet. 2021;12:788. Available from: https://www.frontiersin.org/article/10.3389/fgene.2021.673286 https://doi.org/10.3389/fgene.2021.673286

22. Knott GJ, Doudna JA. CRISPR-Cas guides the future of genetic engineering. 2018;361(6405):866-9. https://doi.org/10.1126/science.aat5011

23. Yin H, Kanasty RL, Eltoukhy AA, Vegas AJ, Dorkin JR, Anderson DG. Non-viral vectors for gene-based therapy. Nat Rev Genet [Internet] 2014;15(8):541-55. https://doi.org/10.1038/nrg3763

24. Wang M, Glass ZA, Xu Q. Non-viral delivery of genome-editing nucleases for gene therapy. Gene Ther 2017;24(3):144-50. https://doi.org/10.1038/gt.2016.72

25. Qiu M, Glass Z, Xu Q. Nonviral Nanoparticles for CRISPR-Based Genome Editing: Is It Just a Simple Adaption of What Have Been Developed for Nucleic Acid Delivery? Biomacromolecules 2019;20(9):3333-9. https://doi.org/10.1021/acs.biomac.9b00783 
26. Wang M, Zuris JA, Meng F, Rees H, Sun S, Deng P, et al. Efficient delivery of genome-editing proteins using bioreducible lipid nanoparticles. Proc Natl Acad Sci U S A 2016;113(11):2868-73. https://doi.org/10.1073/pnas.1520244113

27. Finn JD, Smith AR, Patel MC, Shaw L, Youniss MR, van Heteren J, et al. A Single Administration of CRISPR/Cas9 Lipid Nanoparticles Achieves Robust and Persistent In Vivo Genome Editing. Cell Rep 2018;22(9):2227-35. https://doi.org/10.1016/j.celrep.2018.02.014

28. Jiang C, Mei M, Li B, Zhu X, Zu W, Tian Y, et al. A non-viral CRISPR/Cas9 delivery system for therapeutically targeting HBV DNA and PCSK9 in vivo. Cell Res 2017;27(3):440-3. https://doi.org/10.1038/cr.2017.16

29. Cheng Q, Wei T, Farbiak L, Johnson LT, Dilliard SA, Siegwart DJ. Selective organ targeting (SORT) nanoparticles for tissuespecific mRNA delivery and CRISPR-Cas gene editing. Nat Nanotechnol 2020;15(4):313-20.https://doi.org/10.1038/s41565020-0669-6

30. Liu J, Chang J, Jiang Y, Meng X, Sun T, Mao L, et al. Fast and Efficient CRISPR/Cas9 Genome Editing In Vivo Enabled by Bioreducible Lipid and Messenger RNA Nanoparticles. Adv Mater 2019;31(33):1-7. https://doi.org/10.1002/adma.201902575

31. Fodor G. Primary prevention of CVD: treating dyslipidaemia. BMJ Clin Evid 2011;83(10). https://www.aafp.org/afp/2011/0515/p1207.htm

32. Su X, Kong Y, Peng D. Evidence for changing lipid management strategy to focus on non-high density lipoprotein cholesterol. Lipids Health Dis 2019;18(1):1-7. https://doi.org/10.1186/s12944-019-1080-x

33. Handelsman Y, Lepor NE. PCSK9 inhibitors in lipid management of patients with diabetes mellitus and high cardiovascular risk: A review. J Am Heart Assoc 2018;7(13). https://doi.org/10.1161/JAHA.118.008953

34. Chaudhary R, Garg J, Shah N, Sumner A. PCSK9 inhibitors: A new era of lipid lowering therapy. World J Cardiol 2017;9(2):76. https://doi.org/10.4330/wjc.v9.i2.76

35. Wong ND, Jauhiainen MS, Ray KK, Nishikido T. Non-antibody Approaches to Proprotein Convertase Subtilisin Kexin 9 Inhibition: siRNA, Antisense Oligonucleotides, Adnectins, Vaccination, and New Attempts at Small-Molecule Inhibitors Based on New Discoveries. Front Cardiovasc Med | www.frontiersin.org [Internet] $2019 ; 1$. https://doi.org/10.3389/fcvm.2018.00199

36. Wei T, Cheng Q, Min YL, Olson EN, Siegwart DJ. Systemic nanoparticle delivery of CRISPR-Cas9 ribonucleoproteins for effective tissue specific genome editing. Nat Commun [Internet] 2020;11(1):1-12. Available from: http://dx.doi.org/10.1038/s41467-020-17029-3 https://doi.org/10.1038/s41467-020-17029-3

37. Chadwick AC, Musunuru K. Treatment of Dyslipidemia Using CRISPR/Cas9 Genome Editing. Curr Atheroscler Rep 2017;19(7):1-10. https://doi.org/10.1007/s11883-017-0668-8

38. Ruscica M, Zimetti F, Adorni MP, Sirtori CR, Lupo MG, Ferri N. Pharmacological aspects of ANGPTL3 and ANGPTL4 inhibitors: New therapeutic approaches for the treatment of atherogenic dyslipidemia. Pharmacol Res [Internet] 2020;153(January):104653. https://doi.org/10.1016/j.phrs.2020.104653

39. Tikka A, Jauhiainen $M$. The role of ANGPTL3 in controlling lipoprotein metabolism. Endocrine $2020 ; 52$. https://doi.org/10.1007/s12020-015-0838-9

40. Sharma G, Blumenthal RS, Martin SS. Angiopoietin-like 3 ( ANGPTL3 ) - A Novel Therapeutic Target for Treatment of Hyperlipidemia. 2021;3:6-10. https://www.acc.org/latest-in-cardiology/articles/2021/01/06/13/01/angiopoietin-like-3-angptl3

41. Rhee JW, Wu JC. In vivo genome editing of ANGPTL3: a potential therapeutic strategy for coronary atherosclerosis? Nat Rev Cardiol 2018;15(5):259-60. https://doi.org/10.1038/nrcardio.2018.38

42. Long C, Long C, Amoasii L, Mireault AA, Mcanally JR, Li H, et al. Science-2015-Long-science.aad5725. 2015. https://doi.org/10.1126/science.aad5725

43. Wei T, Cheng Q, Min Y-L, Olson EN, Siegwart DJ. Systemic nanoparticle delivery of CRISPR-Cas9 ribonucleoproteins for effective tissue specific genome editing. Available from: https://doi.org/10.1038/s41467-020-17029-3 https://doi.org/10.1038/s41467-020-17029-3

44. Furgurson M, Lagor WR. CRISPR: a promising tool for lipid physiology and therapeutics. 2019;Available from: www.colipidology.com https://doi.org/10.1097/MOL.0000000000000591

45. Ma L, Wen L, Zhao C, Sun W, Liang Y, Duan L, et al. Article 673286 (2021) Nanoparticle Delivery of CRISPR/ Cas9 for Genome Editing. Front Genet | www.frontiersin.org [Internet] 2021;1:673286. https://doi.org/10.3389/fgene.2021.673286

46. Chin JS, Chooi WH, Wang H, Ong W, Leong KW, Chew SY. Scaffold-mediated non-viral delivery platform for CRISPR/Cas9-based genome editing. Acta Biomater 2019;90:60-70.https://doi.org/10.1016/j.actbio.2019.04.020

47. Chen F, Alphonse M, Liu Q. Strategies for nonviral nanoparticle-based delivery of CRISPR/Cas9 therapeutics. Wiley Interdiscip Rev Nanomedicine Nanobiotechnology 2020;12(3):1-14. https://doi.org/10.1002/wnan.1609

48. Chen X, Gonçalves MAFV. Engineered viruses as genome editing devices. Mol Ther 2016;24(3):447-57. https://doi.org/10.1038/mt.2015.164

49. Kotterman MA, Chalberg TW, Schaffer D V. Viral Vectors for Gene Therapy: Translational and Clinical Outlook. Annu Rev Biomed Eng 2015;17:63-89. https://doi.org/10.1146/annurev-bioeng-071813-104938

50. Doudna JA, Charpentier E. Genome editing. The new frontier of genome engineering with CRISPR-Cas9. Science 2014;346(6213):1258096. https://doi.org/10.1126/science.1258096

51. Hajj KA, Whitehead KA. Tools for translation: Non-viral materials for therapeutic mRNA delivery. Nat Rev Mater [Internet] 2017;2(17056):1-17. Available from: http://dx.doi.org/10.1038/natrevmats.2017.56 https://doi.org/10.1038/natrevmats.2017.56 
52. Xu X, Wan T, Xin H, Li D, Pan H, Wu J, et al. Delivery of CRISPR/Cas9 for therapeutic genome editing. J Gene Med 2019;21(7):1-18. https://doi.org/10.1002/jgm.3107

53. Liu C, Zhang L, Liu H, Cheng K. Delivery strategies of the CRISPR-Cas9 gene-editing system for therapeutic applications. 2017;Available from: http://dx.doi.org/10.1016/j.jconrel.2017.09.012 https://doi.org/10.1016/j.jconrel.2017.09.012

54. Yin H, Song C-Q, Dorkin JR, Zhu LJ, Li Y, Wu Q, et al. Therapeutic genome editing by combined viral and non-viral delivery of CRISPR system components in vivo. Nat Biotechnol [Internet] 2016;34(3):328-33. https://doi.org/10.1038/nbt.3471

55. Rosenblum D, Gutkin A, Kedmi R, Ramishetti S, Veiga N, Jacobi AM, et al. CRISPR-Cas9 genome editing using targeted lipid nanoparticles for cancer therapy [Internet]. 2020. Available from: http://advances.sciencemag.org/ https://doi.org/10.1126/sciadv.abc9450

56. Kulkarni JA, Cullis PR, Van Der Meel R. Lipid Nanoparticles Enabling Gene Therapies: From Concepts to Clinical Utility. Nucleic Acid Ther 2018;28(3):146-57. https://doi.org/10.1089/nat.2018.0721

57. Schaefer KA, Wu W-H, Colgan DF, Tsang SH, Bassuk AG, Mahajan VB. Unexpected mutations after CRISPR-Cas9 editing in vivo. Nat Methods [Internet] 2017;14(6):547-8. https://doi.org/10.1038/nmeth.4293

58. Jarrett KE, Lee C, De Giorgi M, Hurley A, Gillard BK, Doerfler AM, et al. Somatic Editing of Ldlr With Adeno-Associated Viral-CRISPR Is an Efficient Tool for Atherosclerosis Research. Arterioscler Thromb Vasc Biol 2018;38(9):1997-2006. https://doi.org/10.1161/ATVBAHA.118.311221

59. Chadwick AC, Musunuru K. CRISPR-Cas9 Genome Editing for Treatment of Atherogenic Dyslipidemia. Arterioscler Thromb Vasc Biol 2018;38(1):12-8. https://doi.org/10.1161/ATVBAHA.117.309326

60. Guo Q, Feng X, Zhou Y. PCSK9 Variants in Familial Hypercholesterolemia: A Comprehensive Synopsis. Front Genet 2020;11(September):1-13. https://doi.org/10.3389/fgene.2020.01020

61. Ding Q, Strong A, Patel KM, Ng S-L, Gosis BS, Regan SN, et al. Permanent alteration of PCSK9 with in vivo CRISPR-Cas9 genome editing. Circ Res 2014;115(5):488-92. https://doi.org/10.1161/CIRCRESAHA.115.304351

62. Chadwick AC, Wang X, Musunuru K. In Vivo Base Editing of PCSK9 (Proprotein Convertase Subtilisin/Kexin Type 9) as a Therapeutic Alternative to Genome Editing. Arterioscler Thromb Vasc Biol 2017;37(9):1741-7. https://doi.org/10.1161/ATVBAHA.117.309881

63. Nishiga M, Qi LS, Wu JC. Therapeutic genome editing in cardiovascular diseases. Adv Drug Deliv Rev 2020;168:147-57. https://doi.org/10.1016/j.addr.2020.02.003

64. Wang X, Musunuru K. Angiopoietin-Like 3: From Discovery to Therapeutic Gene Editing. JACC Basic to Transl Sci 2019;4(6):755-62. https://doi.org/10.1016/j.jacbts.2019.05.008

65. Chadwick AC, Evitt NH, Lv W, Musunuru K. Reduced blood lipid levels with in vivo CRISPR-Cas9 base editing of ANGPTL3. Circulation 2018;137(9):975-7. https://doi.org/10.1161/CIRCULATIONAHA.117.031335 